\title{
Structure of microprocessor-based automation system of oil pumping station "Alexndrovskaya"
}

\author{
Margarita A. Dmitriyenko, Pavel A. Strizhak \\ National Research Tomsk Polytechnic University, 634050 Tomsk, Russia
}

\begin{abstract}
Structure of microprocessed-based automation system (MBAS) of oil pumping station (OPS) «Alexandrovskaya», located on the territory of Tomsk region and forming part of the Oil Transporting Joint Stock Company «Transneft», developed in accordance with the requirements of the guidance document «Complex of the typical design choices automation of OPSs and crude storages on the basis of modern standard solutions and components».
\end{abstract}

\section{Introduction}

Improving the efficiency and reliability of the manufacturing machinery of oil pumping station possible through the use of microprocessor-based automation systems, implementing adaptive algorithms of logic control [1]. Microprocessor-based automation systems of oil pumping station are designed for monitoring, protection and control of manufacturing machinery, maintaining of its optimum operation mode, also they variation to the computer workstation of the operator.

The purpose of this work is to create a block schematic diagram of MBAS, performing the functions of automatic control technological parameters, automatic protection of manufacturing machinery for emergency and limit values of monitored parameters, program management and maintenance of the standard operation mode of manufacturing machinery, display and record the main controllable technological parameters, preparation and transmission of information processing results in the supervisory control system.

\section{The main part}

Block schematic diagram of OPS «Alexandrovskaya» is shown in Figure 1.

MBAS has a distributed structure with central reserved processor that provides a centralized collection of data from process interface unit (PIU), as well as processing and centralized execution of algorithm of control and management all the equipment of the oil pumping station.

Microprocessed-based automation system has three-level-structure [2].

The lower level includes the primary facilities of measuring and gages of technological parameters, local indicating instruments, equipment for local control and signaling. All equipment on the lower level is located directly on the technological object or close to it (on the instrument boards or racks). Shutoff and control valves have electric drives and control units connected to the MBAS in the digital communication channel.

Control board of secondary instruments is meant for installation of measuring devices, display, control and storage the parameters of vibration. To communicate with control board of secondary instruments used fieldbus Modbus (console log Modbus RTU).

Middle level includes a control board of hand control group, control board of the central controller, control boards of process interface units (PIU 5.1, PIU 5.2, PIU 6.1, PIU 7.1). The control board of the central controller is composed of two central controller with the function of the hot backup, implemented on the basis of modules Modicon Quantum corporation «Schneider Electric», as well as equipment for communication console log Modbus TCP with servers of input/output, computer workstation of the operator and process interface units [3].

Control boards of process interface units also implemented on the basis of controllers Modicon Quantum. PIU 5.1 editorial includes the Programmable Login Controller (PLC) ELSY-TM serve as a communications controller. To ensure the reliable data exchange, as well as high speed data transfer between the PLC uses a high-performance network RIO topology "duplicate ring". PIU 7.1 are located far away from the control board of the central controller. For the exchange of information between them using a duplicate optical link. Control board of hand control group is meant for command issuing bypassing microprocessor automation facilities: emergency shutdown OPS («Stop OPS»); shutdown of booster pump unit (BPU 1, BPU, 2, BPU, 3); management slide-valves of linking-up BPU (№ 7.1.1, № 7.1.2, № 7.1.3). Buttons of control board of hand control group directly affect the control system of high voltage switch unit pump with a delivery of signals instructing the microprocessor system automation. In addition the control board of hand control group includes light alarm. Panel PC, which is a part of the control board of hand control group, connected to a central controller using technologic network Ethernet. By way of guaranteed source of power supply for the medium-level MBAS provides two uninterruptive power supplies (UPS 1, UPS 2).

\footnotetext{
${ }^{\mathrm{a}}$ Corresponding author: mad2@tpu.ru
} 

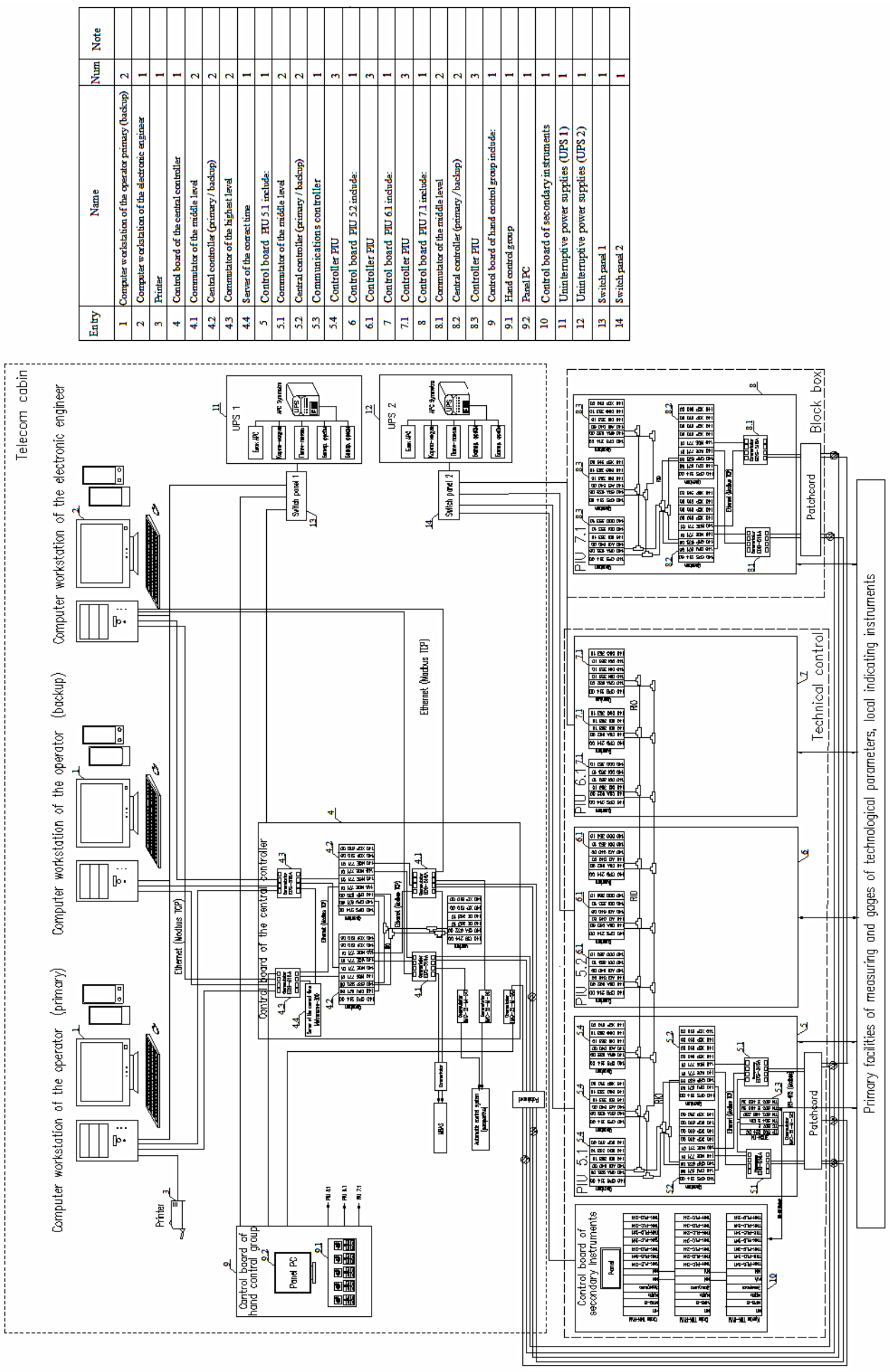

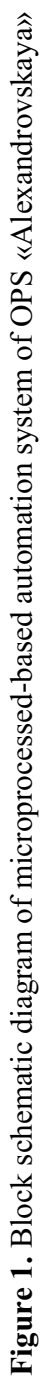


The highest level includes the computer workstation of the operator (primary, backup), combined with the servers of input/output, computer workstation of the electronic engineer (system of diagnostics MBAS), laptop for allowing the configuration data upload to the control boards of process interface units, a printer and other peripheral equipment. The highest level provide to receive and display information from the middle level, monitoring and operations management of the technological process, the archivation events in the system and the actions of the operator OPS, supervision and work management of MBAS OPS to the telemechanic channels.

\section{Conclusions}

Technical solutions embodied in the MBAS OPS «Alexandrovskaya» ensure meeting the requirements of the Oil Transporting Joint Stock Company «Transneft» to control and management the technological process of transportation of liquid hydrocarbons through pipe lines.

Research was performed by a grant from the Russian Science Foundation (project №14-39-00003).

\section{References}

1. Gubeev T.N. Opportunities for improving microprocessor automation systems // Transport and storage of petroleum products and hydrocarbon materials. - 2009. - № 4-5. - P. 16-19.

2. Chirikov S.V., Filippov S.V., Obgoltc A.A., Khromov I.G. Experience in automation of oil pumping stations // Safety in Industry. - 2001. - № 6. - P. 6-8.

3. Automation of oil pumping stations on the basis of controllers Modicon TSX Quantum // Industrial Automation. - 2003. - № 6. - P. 18-20. 